\title{
Assessment of the current drinking pattern in Poland. A study among people hospitalised at the Institute of Psychiatry and Neurology in Warsaw
}

\author{
Andrzej Silczuk', Witold A. Zatoński ${ }^{2,3}$ \\ 'Institute of Psychiatry and Neurology in Warsaw, Poland \\ ${ }^{2}$ Health Promotion Foundation, Nadarzyn, Poland \\ ${ }^{3}$ European Observatory of Health Inequalities, the President Stanisław Wojciechowski State University of Applied Sciences \\ in Kalisz, Poland
}

\begin{abstract}
This paper presents an outline of a research project that will be carried out in 2020 in the Detoxification Ward and the Rehabilitation Therapy Ward of the Department of Prevention and Treatment of Addictions of the Institute of Psychiatry and Neurology in Warsaw, Poland. The cross-sectional study will describe the population of alcohol-dependent people and characterise the dominant drinking pattern in this population. In particular, the frequency of alcohol use and preferences in terms of volume of alcohol bottles will be assessed. An additional objective of the study will be to compare the population of people hospitalised for alcohol withdrawal syndromes with people hospitalised for rehabilitation therapy. The hypothesis will be interrogated that an increase in demand for small vodka bottles (SVBs) correlates with the change in the current pattern of alcohol drinking. So far the drinking pattern in Poland has been described as a pattern of heavy drinking - so-called binge drinking. With growing interests of consumers in SVBs there is a high probability of the appearance of a new pattern, constituting a model in which the patient drinks alcohol in small portions several times a day, maintaining a relatively stable blood alcohol concentration. In recent decades an increased alcohol consumption per capita per year in Poland and mortality rates due to alcohol use have been observed. Therefore, if confirmed by the study, this new emerging drinking pattern may become one of the main tasks and challenges for Public Health.
\end{abstract}

KEY WORDS: alcohol, drinking pattern, alcohol-related diseases.

ADDRESS FOR CORRESPONDENCE: Dr Andrzej Silczuk, Institute of Psychiatry and Neurology in Warsaw,

9 Sobieskiego St., 02-957 Warsaw, Poland, e-mail: asilczuk@ipin.edu.pl

\section{INTRODUCTION}

For the past two decades we have been documenting the scale, health impact, and economic underpinnings of alcohol consumption in Poland. Our research has shown that alcohol is a leading contributor to Poland's high levels of premature mortality, which remains among the highest in the European Union [1-12]. It is particularly concerning that no improvement has been observed in this period in terms of alcohol-related harm. On the contrary, since 2003 we have seen a rapid increase in alcohol consumption in Poland, from 6.5 to 10.2 litres of pure alcohol per capita of the adult population per year. At the same time, patterns of drinking have been changing. Beer has replaced vodka as the most consumed type of alcohol, although hard spirits still account for about one-third of all alcohol consumed. More recently, we have seen indications that the traditional dominant pattern of alcohol consumption - "binge drinking" (sessions of drinking large amounts of alcohol at one event) - has been increasingly replaced by the consumption of smaller portions of alcohol with shorter intervals between drinks, but throughout the whole day. We have described the accompanying rapid rise in the sale of small vodka bottles (SVBs) in a recent publication [7]. 
Despite the significant magnitude and consequences of the described phenomenon, there are almost no serious studies and scientific publications analysing it. These rapid changes, and this gap in literature, constitute the background of the study presented in this article.

\section{AIM OF THE STUDY}

The study will be a cross-sectional description of the population of alcohol-dependent people (hospitalised at the Institute of Psychiatry and Neurology in Warsaw), characterising the dominant drinking pattern in this population. In particular, the frequency of alcohol use and preferences in terms of volume of alcohol bottles will be assessed. An additional objective of the study will be to compare the population of people hospitalised for alcohol withdrawal syndromes with people hospitalised for rehabilitation therapy. While most previous studies of alcohol consumption patterns have been conducted on populations predominantly representative of the general population, so far very few studies have been conducted on the population of Polish addicts treated in hospitals. In one recent study in this population no correlation was found between the damage and the length of alcohol consumption or daily alcohol intake [13].

\section{MATERIAL AND METHODS}

The project will be carried out in the year 2020 in the Detoxification Ward and the Rehabilitation Therapy Ward of the Department of Prevention and Treatment of Addictions of the Institute of Psychiatry and Neurology in Warsaw, Poland. This research project obtained the consent of the local Bioethics Commission no. 21/2019. The following hypotheses have been formulated for the study.

1. In the current population of people hospitalised in the Institute of Psychiatry and Neurology in Warsaw due to alcohol dependence, SVBs are preferred to larger volume bottles.

2. People who drink alcohol several times a day in small portions are more often hospitalised in the detoxification ward than in the rehabilitation unit.

3. People who drink alcohol several times a day in small portions have more often used psychoactive substances other than alcohol in the past.
4. General health based on laboratory tests and clinical observations of people who drink alcohol several times a day in small portions is worse than those who engage in binge drinking episodes.

A minimum of 200 patients will be included in this study, including at least 100 patients hospitalised for alcohol withdrawal syndrome (AWS) at the detoxification ward and at least 100 patients treated for addiction in the rehabilitation ward. The sample size was established on the minimum of 138 subjects, which allows us to provide power calculations and conclusions. For this research model, it is assumed that people hospitalised in the detoxification ward are patients with less intrinsic motivation to undergo addiction treatment but with greater damage. People treated in the rehabilitation therapy ward have greater internal motivation to undergo treatment and are probably less damaged. Both groups represent patients suffering from an alcohol use disorder on a clinically significant level. The inclusion and exclusion criteria formulated in the study are shown in Table 1. The study will not receive any financial support and will be funded by the Institute of Psychiatry and Neurology in Warsaw only.

Patients positively qualified to participate in this study, after giving informed consent, will be assessed using questionnaire methods. Data will be collected using the AUDIT test and a simple survey (tailored for use in this study) containing questions on demographic data, data on the type of alcohol consumed, the frequency of its use, volume of alcohol bottle used, the length of the last draft, the course of the current disease and attempted treatment, and addictive substances besides alcohol. The results of laboratory tests, in particular: blood morphology, AST, ALT activity, GGTP, urea, creatinine, serum amylase, and ionogram, will be compared within the group of people hospitalised in the detoxification ward in subgroups of drinkers in the "binge drinking" pattern and drinking pattern of more often with smaller portions. Blood tests are part of the routine packet of screening tests used to admit patients to the hospital's detoxification department. The same comparison will be provided to patients admitted to the rehabilitation therapy ward. The collected data will be statistically processed. Research results, demographic data, and other quantitative data will be collected and analysed using IBM SPSS Statis-

TABLE 1. Inclusion and exclusion criteria for the study

\begin{tabular}{|l|l|}
\hline Inclusion criteria & $\begin{array}{l}\text { - Age 18-65 years } \\
\text { - Informed consent to participate in the study } \\
\text { - Fulfilled criteria of alcohol dependence according to ICD-10 research criteria for F10.2 }\end{array}$ \\
\hline Exclusion criteria & $\begin{array}{l}\text { - Diagnosis of current use or dependence of drugs such as benzodiazepines, non-benzodia- } \\
\text { zepine hypnotics, opioids, and medicines registered as drugs in the treatment of alcohol } \\
\text { dependence } \\
\text { - Inability to participate in the study due to mental state (disorder of consciousness, significant } \\
\text { cognitive impairment, inability to collect reliable data) }\end{array}$ \\
\hline
\end{tabular}


tics. Appropriate statistical tests in subsequent analysis steps will be chosen on basis of the results of tests for compliance with the normal distribution (Kolmogorow-Smirnov and/or Shapiro-Wilk). To check the intergroup differences, parametric Student's t-test for independent samples will be used. If a statistically significant result is obtained $(p<0.05)$, the value of the effect force will be calculated. Relationships between two variables expressed on a nominal or ordinal scale will be tested using the $\chi^{2}$ test. For statistically significant results, the strength value of this relationship will be additionally calculated by the contingency coefficient $\mathrm{C}$.

\section{EXPECTED RESULTS AND THEIR SIGNIFICANCE}

The goal of this study is to deepen the knowledge in the field of alcohol dependence, in particular in the population of people hospitalised due to alcohol use. A comparison of groups of people hospitalised for alcohol withdrawal in the detoxification ward and for alcohol dependence in the rehabilitation therapy ward will be provided. The results of this study will allow researchers to verify the hypotheses that until now have not been supported by previous studies. The results and conclusions of this study may become the basis for utilitarian applications for the construction of preventive programs in the future, and may have a possible impact on legislation concerning health policy and resulting in a less harmful model of drinking alcohol.

\section{DISCUSSION}

For the purposes of social policy interventions in the field of prevention of substance abuse and dependencies, it is vital to monitor current drinking patterns. Drinking patterns are shaped by many of the complex elements that make up the way of drinking alcohol. These include such elements as: who drinks alcohol (age, sex, health), where users drink alcohol (at home, in bars, restaurants, or other public places), and when users drink alcohol (at meals, at meetings, in free time). Other key factors include types of alcohol consumed (for example: high-quality industrially produced alcoholic beverages or moonshine), how these drinks are consumed (e.g. while enjoying a drink with a meal or drinking to get drunk), and how users drink (whether consumption takes place at one sitting or is spread over a longer period of time). So far, the direct and indirect effects of increased demand for SVBs have not been studied and are not well known. The hypothesis may be raised that an increase in demand for small-volume, high-percentage alcohol bottles correlates with the change in the current model of alcohol drinking. So far, drinking patterns in Poland have been described as a model of heavy drinking - so-called binge drinking [14-16]. With growing interest of consumers in SVBs, there is a high probability of the appearance of a new pattern, constituting a model in which the patient drinks alcohol in small portions several times a day, maintaining a relatively stable blood alcohol concentration (BAC). The observed $40 \%$ increase of pure (100\%) alcohol consumption per capita per year in Poland obliges scientific and clinical communities to perform research and intervene. A yearly increase in mortality rates attributable to alcohol of 6.64 deaths per 100,000 in men aged 45-64 years has been reported [11]. Because about $25 \%$ of premature deaths of adult Polish citizens is related to alcohol [12], to assess a possible new drinking model may be one of the key tasks and challenges for Public Health.

\section{DISCLOSURE}

The authors report no conflict of interest.

\section{References}

1. Rehm J, Sulkowska U, Mańczuk M, et al. Alcohol accounts for a high proportion of premature mortality in central and eastern Europe. Int J Epidemiol 2007; 36 (2): 458-467.

2. Świątkiewicz G, Wieczorek $€$, Allamani A. What influences changes in alcoholic beverage consumption over time? Poland in the light of the European Union Amphora Study. Subst Use Misuse 2014; 49 (12): 1601-1610.

3. Mackenbach JP, Kulhanova I, Bopp M, et al. Inequalities in alcohol-related mortality in 17 European countries: a retrospective analysis of mortality registers. PLoS Med 2015; 12 (12): e1001909.

4. Popova S, Rehm J, Patra J, Zatoński W. Comparing alcohol consumption in central and eastern Europe to other European countries. Alcohol Alcohol 2007; 42 (5): 465-473.

5. Rehm J, Stelemekas M, Badaras R. Research protocol to evaluate the effects of alcohol policy changes in Lithuania. Alcohol Alcohol 2019; 54 (1): 112-118.

6. Rehm J, Zatoński W, Taylor B, Anderson P. Epidemiology and alcohol policy in Europe. Addiction 2011; 106 (Suppl 1): 11-19.

7. Zatoński WA, Młoźniak I, Zatoński M, Gruszczyński Ł. Small bottles - huge problem? A new phase of Poland's ongoing alcohol epidemic. J Health Inequal 2019; 5 (1): 86-88.

8. Zatoński W and the HEM project team. Closing the health gap in European Union. The Maria Skłodowska-Curie Memorial Cancer Center and Institute of Oncology, Warsaw 2008.

9. Lachenmeier DW, Ganss S, Rychlak B, et al. Association between quality of cheap and unrecorded alcohol products and public health consequences in Poland. Alcohol Clin Exp Res 2009; 33 (10): 1757-1769.

10. Stokłosa M, Drope J, Zatoński M, Zatoński WA. Towards improved public health: Affecting alcohol and tobacco affordability and consumption in Poland through taxation. J Health Inequal 2016; 2 (2): 101-104.

11. Zatoński W, Sulkowska U, Zatoński M, et al. Alcohol taxation and premature mortality in Europe. Lancet 2015; 385 (9974): 1181.

12. Zatoński WA. One hundred years of health in Poland. J Health Inequal 2019; 5 (1): 11-19.

13. Silczuk A, Habrat B, Lew-Starowicz M. Thrombocytopenia in patients hospitalized for Alcohol Withdrawal Syndrome and its 
associations to clinical complications. Alcohol Alcohol 2019; 54 (5): 503-509.

14. Moskalewicz, J. Privatization of the alcohol arena in Poland Contemporary Drug Problems 1993; 20: 263-275.

15. Cherpitel CJ, Moskalewicz J, Swiatkiewicz G. Drinking patterns and problems in emergency services in Poland. Alcohol Alcohol 2004; 39 (3): 256-261.

16. Anderson P, Møller L, Galea G. Alcohol in the European Union. Consumption, harm and policy approaches. WHO Regional Office for Europe, Copenhagen 2012.

\section{AUTHORS' CONTRIBUTIONS}

AS prepared research concept and design of the publication and wrote the article. WAZ critically revised it. Both authors contributed equally to the final version of publication. 